\title{
Emphysematous Pyelonephritis and Emphysematous Osteomyelitis: A Case Report
}

\author{
Sanae Sninate ${ }^{1,2^{*}}$, Tlaite Oubaddi ${ }^{1,2}$, Soukaina Allioui ${ }^{1,2}$, Leila Jroundi ${ }^{1,2}$, \\ Fatima Zahrae Laamrani ${ }^{1,2}$
}

\begin{abstract}
Background. Emphysematous osteomyelitis and emphysematous pyelonephritis are both rare entities responsible for a high death rate. The first is defined by the presence of intravertebral or intraosseous gas, while the second is infectious damage to the renal parenchyma and perilesional tissues caused by gas-producing microorganisms and is, therefore, characterized by the formation of gas. Imaging plays a crucial role in making a rapid diagnosis and, thus, the initiation of the necessary treatment.

Case Report. A 66-year-old woman with a medical history of type 2 diabetes mellitus and hypertension presented with the altered general condition and flank pain for 5 days, associated with fever and chills. The patient's vital signs were remarkable with a disturbed biological workup, which led to emergency abdominal and pelvic computed tomography scanning that revealed emphysematous osteomyelitis of the spine and emphysematous pyelonephritis.

Conclusions. The coexistence of emphysematous pyelonephritis and emphysematous osteomyelitis is a rare life-threatening entity, occurring in patients with comorbidities such as diabetes mellitus. Computed tomography is currently the gold standard in making the positive diagnosis, staging, for a quick and better management and, thus, a favorable prognosis.
\end{abstract}

\section{Keywords}

Emphysematosus Osteomyelitis; Emphysematosus Pyelonephritis; Imaging

${ }^{1}$ Emergency Radiology Department, Ibn Sina Hospital, Mohammed V University, Rabat, Morocco

${ }^{2}$ Faculty of Medicine and Pharmacy of Rabat, Mohammed V University, Rabat, Morocco

*Corresponding author: sninate.sanae@gmail.com

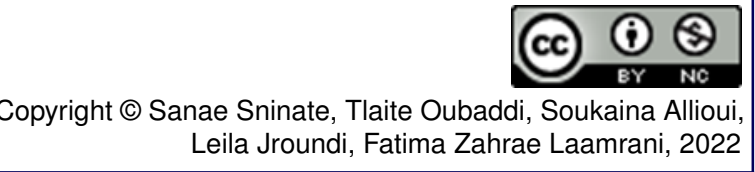

\section{Introduction}

Emphysematous pyelonephritis (EPN) is a necrotizing, gasforming infection of the renal parenchyma and perirenal tissue [1]. The recent mortality rate from EPN is $25 \%$ [2]. The clinic is non-specific; the most common symptoms are fever, flank pain and nausea.

Emphysematous osteomyelitis (EO) is a rare and potentially fatal infection caused by intraosseous gas-forming bacteria [3]. EO was introduced by Ram et al. in 1981 when they detected bubbles of intraosseous gas on a patient's X-ray image [4]. Vertebral osteomyelitis is secondary to hematogenous seeding of germs from the urinary tract.

The coexistence of EPN and EO is a potentially lifethreatening condition. It occurs almost exclusively in patient with diabetes mellitus. If not treated early, it may lead to fulminant sepsis and, therefore, carries a high mortality.

We report a rare case of both EPN and EO in a patient with uncontrolled hyperglycemia by underlining the role and contribution of computed tomography (CT) in the diag- nosis of these entities and the timely initiation of aggressive treatment.

\section{Case Report}

A 66-year-old woman with a medical history of type 2 diabetes mellitus and hypertension presented with the altered general condition and flank pain associated with fever and chills lasting for 5 days. On admission, the patient's vital signs were remarkable, with a fever and temperature of $40^{\circ} \mathrm{C}$, heart rate of $90 \mathrm{bpm}$, blood pressure of $143 / 94 \mathrm{~mm} \mathrm{Hg}$, respiratory rate of $18 \mathrm{bpm}$, and saturation of $99 \%$ at room air. She appeared obese, confused, and agitated with flank tenderness. Laboratory examinations revealed a high white blood cell count $-33 \times 10^{9} / \mathrm{L}(4.0$ $\left.12.0 \times 10^{9} / \mathrm{L}\right)$, creatinine $-9 \mathrm{mg} / \mathrm{dL}(0.60-1.40 \mathrm{mg} / \mathrm{dL})$, total bilirubin $-3 \mathrm{mg} / \mathrm{dL}(0.2-1.2 \mathrm{mg} / \mathrm{dL})$, lipase $-500 \mathrm{U} / \mathrm{L}$ $(0-140 \mathrm{U} / \mathrm{L})$, and glucose $-270 \mathrm{mg} / \mathrm{dl}$. Urinalysis revealed macroalbuminuria $(>300 \mathrm{mg} / \mathrm{dl}$ ). Platelet count was $31 \times 10^{3} / \mu \mathrm{L}$, aminotransferase $-60 \mathrm{U} / \mathrm{L}(10-40 \mathrm{U} / \mathrm{L})$, 
and alanine aminotransferase - $40 \mathrm{U} / \mathrm{L}$ (3-45 U/L). Creactive protein was $420 \mathrm{mg} / \mathrm{L}$. Septic shock was suspected, and the patient was given empiric therapy with piperacillintazobactam and vancomycin.

Abdominal and pelvic CT performed without intravenous (IV) contrast revealed left renal hypertrophy, ureterohydronephrosis with the presence of gas in the calices, infiltration of septa in the perirenal space (Fig. 1), intraosseous gas in the vertebral bodies, D12, L3 and L4 and emphysema in disc space L3-L4, epidural spaces (Fig. 2) concluding in emphysematous pyelonephritis and emphysematous osteomyelitis.

a

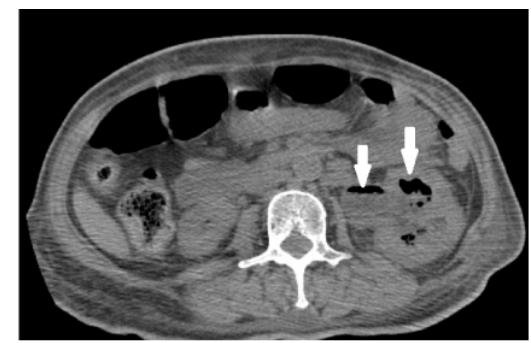

b

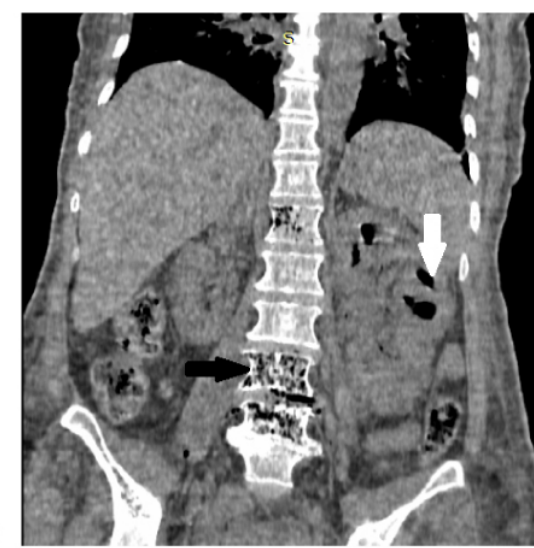

Figure 1. Axial (a) and coronal (b) abdominal CT scans without IV contrast which revealed left renal hypertrophy, ureterohydronephrosis with the presence of air in the calices (white arrow) and infiltration of septa in the perirenal space, intraosseous gas in the vertebral bodies, D12, L3 and L4 (black arrow).

A Foley catheter was placed; blood and urine cultures were performed; a broad-spectrum IV antibiotic therapy with piperacillin-tazobactam and vancomycin was initiated. The blood culture was negative; however, the urine culture revealed the presence of Escherichia coli (10,000$50,000 \mathrm{CFU} / \mathrm{ml}$ ), sensitive to ceftriaxone. The antibiotic dose was reduced to ceftriaxone. The patient's clinical condition improved on the $10^{\text {th }}$ day.

\section{Discussion}

EPN is a severe and life-threatening necrotizing renal and perirenal infection with gas in the renal parenchyma, collecting system, or perinephric tissue [2].

EPN disproportionately affects females, with a femaleto-male ratio of $3: 1$ [5]. The increase in frequency among women may be related to their increased susceptibility to urinary tract infections. The most frequently responsible

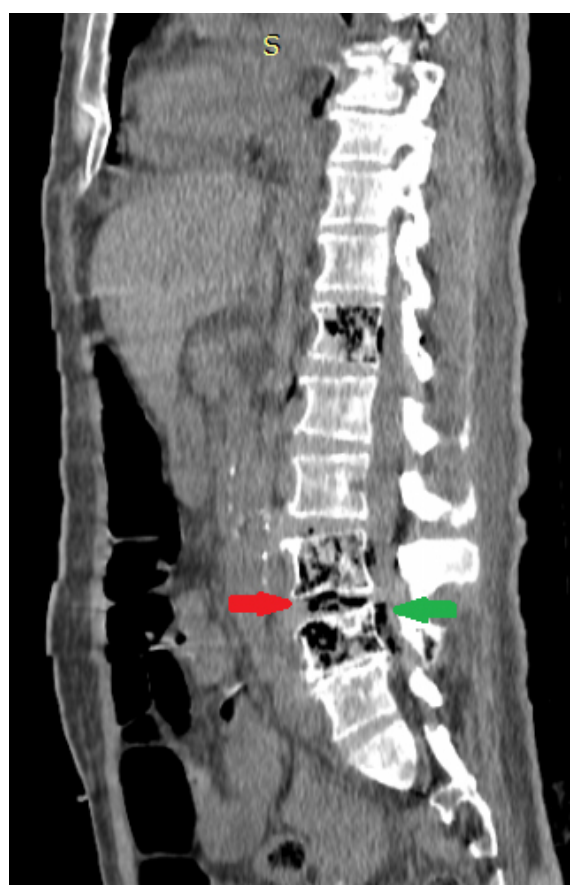

Figure 2. Sagittal abdominal CT scan without IV contrast which showed intraosseous gas within D12, L3 and L4, vertebral bodies and emphysema in disc space L3-L4 (red arrow), epidural space (green arrow).

pathogens are Escherichia coli, Klebsiella pneumoniae, Proteus mirabilis, and Pseudomonas aeruginosa [5].

Clinically, most patients complain of fever, abdominal, flank or back pain consistent with signs of pyelonephritis.

Physical examination for EPN is inconclusive as the retroperitoneal location of the kidneys makes palpation difficult in crepitation. Although palpable soft tissue gas is reported in $12 \%$ of patients. The biological assessment shows leukocytosis and sometimes thrombocytopenia, elevated creatinine levels. Cytobacteriological examination of the urine reveals pyuria in most cases [1].

EO is a rare entity with only 35 prior cases described in the literature [6]. It is characterized by the presence of intraosseous gas. The vertebral bodies, pelvis, and lower limb bones are most often affected [7]. Hematogenous dissemination is the most common route of infection.

Due to the high morbidity and mortality rates, it is necessary to diagnose EO early to start aggressive treatment [3].

Although early diagnosis of EO is critical, there are other differential diagnoses revealed by intraosseous gas. The first and most common is gas in the intervertebral discs, joints, or adjacent bony structures related to degenerative arthritis and trauma. This "vacuum phenomenon" and the pneumatocysts both in the spine and elsewhere in the body have been described [3]. In our case, it was the result of disseminated urinary tract infection that reached the bone, as well as the kidneys.

The radiologist plays a crucial role in the diagnosis of EPN as prompt management is necessary once the case is confirmed. Abdominal X-rays can be helpful in detecting gas pockets in the renal shadow, bone tissue and soft 
tissue, but are still insufficient. Ultrasound can also show an abnormal kidney with nondependent echoes, but there can be misdiagnosis due to the presence of adjacent gas or bowel stones, which can lead to confusion [8]. Abdominal $\mathrm{CT}$ is the most useful and sensitive tool [7]. It allows for more accurate staging of the disease and is considered as the gold standard of diagnosis. It can distinguish the type of EPN [5]. Findings include renal parenchymal enlargement and destruction, presence of gas, fluid collections, gas-fluid levels, focal tissue necrosis and possibly abscess formation.

$\mathrm{CT}$ is the most sensitive method to diagnose EO; conventional X-ray imaging has a low sensitivity rate of detecting intraosseous gas [4].

Treatment must be intensive with vigorous fluid resuscitation, systemic administration of antibiotics, and strict control of blood glucose and careful monitoring of electrolytes. Before the advent of interventional radiology, early surgery and nephrectomy were an important part of the therapeutic treatment plan [9].

EO is associated with high morbidity and mortality of up to $32 \%$, especially in patients with diabetes mellitus. It should, therefore, be treated intensively with antibiotics for 4 to 6 weeks to avoid necrosis and bone destruction. Surgery should be reserved for complicated situations such as abscess formation or necrosis, and for patients who do not respond to antibiotics [10].

\section{Conclusions}

The coexistence of EPN and EO is a rare life-threatening entity, occurring in patients with comorbidities such as diabetes mellitus. CT scan plays a crucial role in making the positive diagnosis, staging, for a quick and better management and, thus, a favorable prognosis.

\section{Ethical Statement}

The research was conducted according to the Declaration of Helsinki.

\section{Informed Consent}

Written informed consent was obtained from the patient who participated in this case.

\section{Conflict of Interest}

The authors declared no conflicts of interest.

\section{Financial Disclosure}

This research received no specific grant from any funding agency in the public, commercial, or not-for-profit sectors.

\section{References}

[1] Koch GE, Johnsen NV. The diagnosis and management of life-threatening urologic infections. Urology. 2021;156:6-15. Available from: https://doi.org/10.1016/j.urology.2021.05.011
[2] Kamei J, Yamamoto S. Complicated urinary tract infections with diabetes mellitus. Journal of Infection and Chemotherapy. 2021;27(8):1131-1136. Available from: https://doi.org/10.1016/j.jiac.2021.05.012

[3] Sax AJ, Morrison WB, Belair JA. Extensive multifocal emphysematous osteomyelitis: fatal outcome in a patient with psychiatric history. Skeletal Radiology. 2020;49(9):1487-1493. Available from: https://doi.org/10.1007/s00256-020-03470-4

[4] Sulyma V, Sribniak A, Bihun R, Sribniak Z. Emphysematous osteomyelitis: review of the literature. Ortopedia, Traumatologia, Rehabilitacja. 2020;22(3):153-159. Available from: https://doi.org/10.5604/01.3001.0014.3231

[5] Tatakis F-P, Kyriazis I, Panagiotopoulou I-E, Kalafatis E, Mantzikopoulos G, Polyzos K, et al. Simultaneous diagnosis of emphysematous osteomyelitis and emphysematous pyelonephritis in a diabetic patient. American Journal of Case Reports. 2019;20:1793-1796. Available from: https://doi.org/10.12659/AJCR.920006

[6] Small JE, Chea P, Shah N, Small KM. Diagnostic features of emphysematous osteomyelitis. Current Problems in Diagnostic Radiology. 2018. Available from: https://doi.org/10.1067/j.cpradiol.2018.05.008

[7] Lee J, Jeong CH, Lee MH, Jeong E-G, Kim YJ, Kim SI, et al. Emphysematous osteomyelitis due to Escherichia coli. Infection \& Chemotherapy. 2017;49(2):151-154. Available from: https://doi.org/10.3947/ic.2017.49.2.151

[8] Craig WD, Wagner BJ, Travis MD. Pyelonephritis: radiologic-pathologic review. RadioGraphics. 2008;28(1):255-276. Available from: https://doi.org/10.1148/rg.281075171

[9] Tang HJ, Li CM, Yen MY, Chen YS, Wann SR, Lin HH, Lee SS, Liu YC. Clinical characteristics of emphysematous pyelonephritis. J Microbiol Immunol Infect. 2001;34(2):125-130. Available from: https://pubmed.ncbi.nlm.nih.gov/11456358/

[10] Mujer MT, Rai MP, Hassanein M, Mitra S. Emphysematous osteomyelitis. BMJ Case Rep. 2018;2018:bcr2018225144. Available from: https://doi.org/10.1136/bcr-2018-225144

Received: 2021-09-14

Revision Requested: 2021-10-11

Revision Received: 2021-10-20

Accepted: 2021-10-23 